\title{
Video Case 2: Polypectomy in Ulcerative Colitis Patient
}

\section{Tarik Zaher \\ Tropical Medicine Department, Faculty of Medicine, Zagazig University, Egypt}

A 58-years old Egyptian women presented by bleeding per rectum for 4 monthes was examined by colonoscopy which revealed inflammation and friability of the mucosa of rectum, sigmoid and descending colon with single pedunculated sigmoid colon polyp.Histopathology of mucosal biopsies confirmed the diagnosis of ulcerative colitis.Histopathology of biopsies from the polyp revealed hyperplastic nature of the
polyp.The patient was treated by oral mesalamin (3 gm per day) and oral azathioprin(100 mg per day) with successful clinical remission. One month later the patient was prepared for polypectomy( see the video) which was followed by mild blood ooze which was secured by band ligation of the stump.This polyp was different from the multiple sessile pseudo-polyps of ulcerative colitis which do not need polypectomy. 